\title{
Sporadic pseudohypoaldosteronism: A challenging diagnosis
}

\author{
Suman Preet Kaur Bhullar, Raouf Seifeldin, Nikhil Hemady
}

\begin{abstract}
Introduction: Pseudohypoaldosteronism (PHA) is a rare form of salt-wasting syndrome, caused by peripheral resistance to aldosterone. PHA is of three types: PHA type 1, 2, 3 . Pseudohypoaldosteronism type 1 (PHA1) is further differentiated into, (i) hereditary forms, autosomal recessive and dominant, which are caused by epithelial sodium channel and mineralocorticoid receptor mutations respectively and (ii) secondary form which is associated with urological problems. Case Report: We present a case of a male infant who presented with failure to thrive, vomiting, mild dehydration and reflux. Evaluation revealed hyperkalemia with normal glucose and carbon dioxide levels. A preliminary diagnosis of CAH (congenital adrenal hyperplasia) was made. Further workup showed high serum aldosterone and renin levels with normal renal and adrenocortical functions. In line with the investigations the diagnosis of pseudohypoaldosteronism was made. The patient was treated with sodium supplementation, which normalized his clinical
\end{abstract}

Suman Preet Kaur Bhullar ${ }^{1}$, Raouf Seifeldin ${ }^{2}$, Nikhil Hemady ${ }^{3}$

Affiliations: ${ }^{1}$ Resident, Department of Family Medicine, Doctors' Hospital of Michigan, Pontiac, MI, USA; ${ }^{2}$ Associate Program Director, Department of Family Medicine, Doctors' Hospital of Michigan, Pontiac, MI, USA; ${ }^{3}$ Program Director, Department of Family Medicine, Doctors' Hospital of Michigan, Pontiac, MI, USA.

Corresponding Author: Suman Preet Kaur Bhullar, MD Resident, Department of Family Medicine, 461 W Huron Street. Doctors' Hospital of Michigan. Pontiac, MI, USA; Ph: +1-248-857-7200; Email: drsbhullar@gmail.com

Received: 09 April 2012

Accepted: 06 May 2012

Published: 01 January 2013 state and serum electrolytes. Follow-up revealed weight gain and improved status. Conclusion: Diagnosis of PHA1 is based on plasma electrolyte assessment, elevated renin activity and aldosterone levels with normal renal function. PHA1 results from a renal or systemic resistance to aldosterone. In our reported patient we suspected a renal form of PHA1, which is a milder form and responded well to treatment with salt supplements. Infants who present with electrolyte imbalance like hyperkalemia, hyponatremia and weight loss should be evaluated for adrenocortical function and need careful management. Though PHA is a group of rare syndromes, a high degree of suspicion along with extensive laboratory workup should be pursued in cases with electrolyte imbalances.

Keywords: Pseudohypoaldosteronism, Congenital Adrenal Hyperplasia, Aldosterone

$* * * * * * * * *$

Bhullar SPK, Seifeldin R, Hemady N. Sporadic pseudohypoaldosteronism: A challenging diagnosis. International Journal of Case Reports and Images 2013;4(1):15-18.

$* * * * * * * * *$

doi:10.5348/ijcri-2013-01-250-CR-4

\section{INTRODUCTION}

Pseudohypoaldosteronism (PHA) is a rare form of salt-wasting syndrome, caused by peripheral resistance to aldosterone. PHA has three types: PHA type 1, 2, and 3 . Pseudohypoaldosteronism type 1 (PHA1) is further differentiated into, (i) hereditary forms - autosomal recessive and dominant, which are caused by epithelial 
sodium channel and mineralocorticoid receptor mutations respectively and (ii) secondary form which is associated with urological problems. PHA being a rare disorder, only about seventy cases have been reported in the literature. We report a case of PHA - which inspite of being a rare disorder, can be potentially lifethreatening and also briefly discuss the types of PHA, diagnosis and treatment.

\section{CASE REPORT}

A 5-week-old Caucasian male infant was admitted to the hospital for evaluation of failure to thrive. He was born at term via cesarean section and weighed $3.07 \mathrm{~kg}$ at birth. He was noted to have mild dehydration in spite of feeding well along with on and off vomiting and some reflux. His weight was $2.96 \mathrm{~kg}$ and physical examination was normal at presentation to the hospital. External genitalia were normal.

Initial laboratory investigations showed hyperkalemia with potassium levels of $5.1 \mathrm{mmol} / \mathrm{L}$, with sodium of $122 \mathrm{mmol} / \mathrm{L}$. Repeat testing later on showed potassium level of $8.1 \mathrm{mmol} / \mathrm{L}$. He was resuscitated with D5 0.45 NS. Further biochemistry revealed low urine and serum osmolality, normal urine sodium and urine potassium levels with trace of reducing substance in the urine. Upper gastrointestinal studies were unremarkable. Results of urine analysis and renal functions were normal. Abdominal ultrasound was negative for renal and adrenal abnormalities. A preliminary diagnosis of $\mathrm{CAH}$ (congenital adrenal hyperplasia) was made and patient was started on hydrocortisone $50 \mathrm{mg} / \mathrm{m}^{2}$, i.e $1.5 \mathrm{mg}$ thrice a day.

Further workup showed serum aldosterone level of $2174 \mathrm{mg} / \mathrm{dL}(10-160 \mathrm{mg} / \mathrm{dL})$, PRA (pre renal activity) of $103.9 \mathrm{ng} / \mathrm{mL} / \mathrm{hr}\left(0.5^{-1.19} \mathrm{mg} / \mathrm{mL}\right)$, serum renin level of $193998 \mathrm{ng} / \mathrm{dL} / \mathrm{hr} \quad(0.29-3.7 \mathrm{ng} / \mathrm{dL} / \mathrm{hr})$, carbon dioxide of $19 \mathrm{mmol} / \mathrm{L}$ (normal levels in brackets). Normal levels were found for serum cortisol, androstenedione, 17-OH progesterone, ACTH, FSH, LH and pregnenolone.

In view of the laboratory results hydrocortisone was discontinued and he was started on oral fludrocortisone $0.1 \mathrm{mg}$ twice daily along with $\mathrm{NaCl}$ supplements. Potassium lowering therapies were also used along with sodium supplementation. Patient responded to the treatment and started gaining weight. $\mathrm{He}$ was discharged home with medications and weighed 3520 gms. On follow-up he was found to be gaining appropriate weight and was kept on oral salt supplements alone. Genetic analysis for PHA in the family was negative.

\section{DISCUSSION}

Pseudohypoaldosteronism is a salt-wasting syndrome due to peripheral resistance to aldosterone. This may be either a primary (mutation of MR or ENaC) or a secondary (infection, uropathy, medication) phenomenon. In all cases, sodium reabsorption and potassium excretion are impaired in the principal cell of the collecting duct. The biological characteristics are hyponatraemia, hyperkalaemia and acidosis [1]. It is thus characterized by three essential features: hyperkalemia, metabolic acidosis and elevated aldosterone concentration with normal glomerular filtration rate (GFR). Volume depletion or hypervolemia, renal salt wasting or retention, hypotension or hypertension and elevated, normal-high or low levels of renin and aldosterone may be observed in the various conditions that result in differentiating this syndrome in three types of PHA.

Pseudohypoaldosteronism type 1: This was first described in 1958 by Cheek and Perry [2]. This rare syndrome starts during the neonatal period with a wide spectrum [1]. PHA1 occurs in two genetic forms, (i) a renal form of autosomal dominant inheritance due to a heterozygous mutation of the mineralocorticoid receptor (MR) gene coding for the mineralocorticoid receptor and, (ii) a severe systemic form of autosomal recessive inheritance due to a mutation of the epithelial sodium channel (EnaC) gene, which is a secondary form usually in association with urinary tract malformation and acute pyelonephritis. Autosomal dominant variant is Renal PHA1; while systemic one due to autosomal recessive inheritance is also known as multiple target organ disorder (MTOD).

Renal PHA1 or early childhood hyperkalemia is probably due to a maturation disorder in the number or function of aldosterone receptors and also in sporadic cases. This form manifests with renal salt loss in infancy and failure to thrive and a gradual improvement with advancing age.

In systemic variant, other organs are involved, such as the sweat glands, salivary glands and colon. The fundamental abnormality in multiple target organ defect (MTOD) PHA1 is a loss-of-function mutation in the alpha or beta subunits of the epithelial sodium channel $(\mathrm{ENaC})$ gene, resulting in defective sodium transport in many organs containing the $\mathrm{ENaC}$ gene, (e.g., kidney, lung, colon, sweat and salivary glands). This amiloridesensitive member of the degenerin/epithelial sodium channel (Deg/ENaC) super family of ion channels is comprised of three homologous units (alpha, beta and gamma) and is expressed in the apical membrane of epithelial cells lining the airway, colon, and distal nephron. ENaC plays an essential role in transepithelial $\mathrm{Na}+$ and fluid balance. PHA1 presents with potential life-threatening salt wasting and failure to thrive in early infancy.

Pseudohypoaldosteronism type 2: This is also known as familial hyperkalemia and hypertension or Gordon syndrome [3, 4]. The classification of this heterogeneous syndrome as PHA is, however, controversial because plasma aldosterone concentrations are highly variable, usually almost normal, and patients respond adequately to mineralocorticoid hormone [5]. The organ involvement and genetic abnormality in pseudohypoaldosteronism type 2 (PHA2) is similar to PHA1. The hallmarks of PHA2 are hypertension, hyperkalemia and correction of 
these abnormalities by low doses of thiazide diuretics $[6,7]$.

Pseudohypoaldosteronism type 3: This is transient and secondary to various pathologies related to kidneys or other organs [7]. Rare cases of major intestinal resection [8] or sweat gland dysfunction associated with excessive loss of sodium [9] have been described as leading to PHA III. However, renal causes are encountered more frequently. Nephropathies such as obstructive uropathy [10] or urinary tract infection [11] are reported as causes of transient aldosterone resistance [7]. The main characteristic of this type of PHA is a decreased GFR.

In all cases of PHA, sodium reabsorption and potassium excretion are impaired in the principle cells of collecting ducts. The biological characteristics are hyponatremia, hyperkalemia and metabolic acidosis. After having excluded pseudohyperkalemia due to hemolysis, the diagnosis may be challenging. If serum chloride is normal while serum sodium has decreased and GFR is not impaired, type- 4 renal tubular acidosis can be ruled out. The normal hormone levels of ACTH, 17-OH progesterone and cortisol allows exclusion of adrenal insufficiency. Finally, high aldosterone and plasma renin levels lead to the diagnosis of PHA [1].

We diagnosed our patient as a case of PHA1 which is characterized by neonatal salt wasting, vomiting, dehydration and failure to thrive. We would like to summarize our observations in the management of this patient. In our case, diagnosis of PHA1 was based on plasma electrolyte assessment, high renin activity, high aldosterone levels, low level of carbon dioxide with normal renal function resulting from a renal or systemic resistance to aldosterone. Normal levels of cortisol, 17-OH progestrone, ACTH and androstenedione excluded the diagnosis of congenital adrenal hyperplasia and other corticoid dysfunctions. By doing upper gastrointestinal studies, upper gastrointestinal abnormalities were excluded. Normal ultrasound of kidneys and adrenals excluded anatomical abnormalities. We suspected the sporadic form of PHA1 in our patient and treated him with sodium supplementation which normalized his clinical state and serum electrolytes. There was good response to sodium chloride supplementation and he was thriving well on follow up.

\section{CONCLUSION}

While evaluating any infant with suspected CAH, one should consider pseudohypoaldosteronism as one of the differential and infants who present with electrolyte imbalance like hyperkalemia, hyponatremia and weight loss should be evaluated for adrenocortical function and need careful management. Genetic analysis should be done as the disease can have a genetic predisposition or may be sporadic. Though PHA is a group of rare syndromes, but a high degree of suspicion along with extensive laboratory workup should be pursued in cases with electrolyte imbalances.
A multidisciplinary team approach including a neonatologist, an endocrinologist, genetic expert and a dietician is essential for evaluation of longitudinal growth and neurological development in PHA patients.

$* * * * * * * * *$

\section{Author Contributions}

Suman Preet Kaur Bhullar - Conception and design, Acquisition of data, Analysis and interpretation of data, Drafting the article, Critical revision of the article, Final approval of the version to be published

Rauf Seifeldin - Conception and design, Acquisition of data, Analysis and interpretation of data, Drafting the article, Critical revision of the article

Nikhil Hemady - Drafting the article, Critical revision of the article, Final approval of the version to be published

\section{Guarantor}

The corresponding author is the guarantor of submission.

\section{Conflict of Interest}

Authors declare no conflict of interest.

\section{Copyright}

(C) Suman Preet Kaur Bhullar et al. 2013; This article is distributed under the terms of Creative Commons Attribution 3.0 License which permits unrestricted use, distribution and reproduction in any means provided the original authors and original publisher are properly credited. (Please see www.ijcasereportsandimages.com /copyright-policy.php for more information.)

\section{REFERENCES}

1. Belot A, Ranchin B, Fichtner C, et al. Pseudohypoaldosteronisms, report on a 10-patient series. Nephrol Dial Transplant 2008 May;23(5):1636-41.

2. Cheek DB, Perry JW. A salt wasting syndrome in infancy. Arch Dis Child 1958;33(169):252-6.

3. Gordon RD, Geddes RA, Pawsey CG, O'Halloran MW. Hypertension and severe hyperkalaemia associated with suppression of renin and aldosterone and completely reversed by dietary sodium restriction. Australas Ann Med 1970;19(4):287-94.

4. Gordon RD. Syndrome of hypertension and hyperkalemia with normal glomerular filtration rate. Hypertension 1986;8(2):93-102.

5. Stokes JB. Disorders of the epithelial sodium channel: Insights into the regulation of extracellular volume and blood pressure. Kidney Int 1999;56(6):2318-33.

6. Disse-Nicodeme S, Achard JM, Desitter I, et al. A new locus on chromosome $12 \mathrm{p} 13.3$ for pseudohypoaldosteronism type II, an autosomal dominant form of hypertension. Am J Hum Genet 2000;67(2):302-10.

7. Olivier Bonny, Bernard C Rossier. Disturbances of $\mathrm{Na} / \mathrm{K}$ Balance: Pseudohypoaldosteronism Revisited. J Am Soc Nephrol 2002;13(9):2399-414. 
8. Vantyghem MC, Hober C, Evrard A, et al. Transient pseudo-hypoaldosteronism following resection of the ileum: normal level of lymphocytic aldosterone receptors outside the acute phase. J Endocrinol Invest 1999;22(2):122-7.

9. Anand SK, Froberg L, Northway JD, Weinberger M, Wright JC. Pseudohypoaldosteronism due to sweat gland dysfunction. Pediatr Res 1976;10(7):677-82.

10. Bülchmann G, Schuster T, Heger A, Kuhnle U, Joppich I, Schmidt H. Transient pseudohypoaldosteronism secondary to posterior urethral valve-A case report and review of the literature. Eur J Pediatr Surg 2001;11(4):277-9.

11. Perez-Brayfield MR, Gatti J, Smith E, Kirsch AJ. Pseudohypoaldosteronism associated with ureterocele and upper pole moiety obstruction. Urology 2001;57(6):1178.
Access full text article on other devices

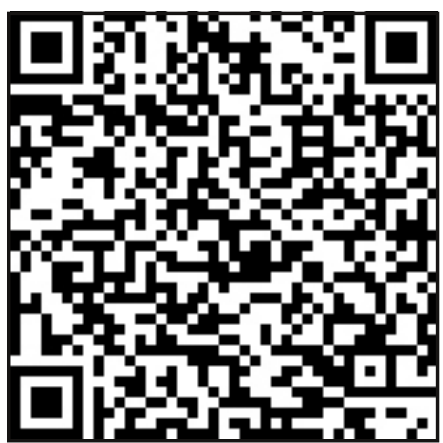

Access PDF of article on other devices

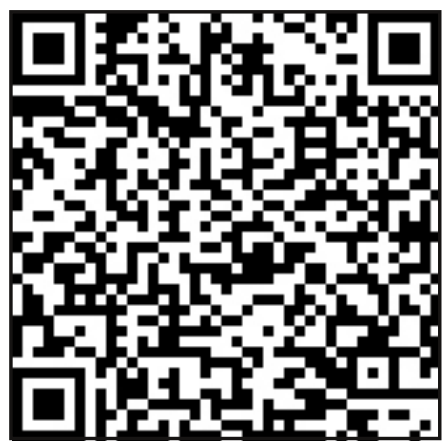

